\title{
FEM-based Soft Robotic Control Framework for Intracavitary Navigation
}

\author{
Kit-Hang Lee ${ }^{1}$, Martin C.W. Leong ${ }^{1}$, Marco C.K. Chow ${ }^{1}$, Hing-Choi Fu ${ }^{1}$, Denny K.C. Fu ${ }^{1}$, \\ Wayne Luk ${ }^{2}$, Fellow, IEEE, Kam-Yim Sze ${ }^{1}$, Chung-Kwong Yeung ${ }^{3}$ and Ka-Wai Kwok ${ }^{1}$
}

\begin{abstract}
Bio-inspired robotic structure composed of sof actuation units has attracted increasing research interests in its potential and capacity of complying with unstructured and dynamic environment, as well as providing safe interaction with human; however, this inevitably poses technical challenging to achieve steady, reliable control due to the remarkable non-linearity of its kinematics and dynamics. To resolve this challenge, we propose a novel control framework that can characterize the kinematics of a soft continuum robot through the hyper-elastic Finiteelement modeling (FEM). This enables frequent updates of the Jacobian mapping from the user motion input to the endeffector's point of view. Experimental validation has been conducted to show the feasibility of controlling the soft robot for intracavitary path following. This could be the first success to demonstrate the perspectives of achieving stable, accurate and effective manipulation under large change of robot morphology without having to deduce its analytical model. It is anticipated to draw further extensive attention on resolving the bottleneck against the application of FEM, namely its intensive computation.
\end{abstract}

Index Terms-Finite-element modeling (FEM), Soft Robot Motion Control

\section{INTRODUCTION}

Recent advances in soft robotics have attracted much research attention. Inspired by nature [1-3], the robot made of soft materials facilitates many applications demanding for safe manipulation interacting with human, as well as excellent compliance with confined, unstructured and dynamic environment. Minimally invasive surgery could be a typical example $[4,5]$. The inherent flexibility theoretically poses infinite degrees of freedom (DoFs). Unlike the conventional robots comprising of rigid linkages between joints, the kinematics and dynamics of soft robot cannot be explicit and well-formulated, but also susceptible to structural error due to its hyper non-linearity.

Many research attempts [6-9] were to deduce the analytical model which simulates the robot kinematics behavior. Analytical modeling is a common approach to formulate the kinematics of small and simple structured robots [10]. However, in particular for high DoFs or hyperelastic robot structure, the complexity of the model will increase tremendously with the actuation dimensionality, and it will be very challenging to formulate a precise

${ }^{1}$ K.H. Lee, Martin C.W. Leong, Marco C.K. Chow, H.C. Fu, Denny K.C. Fu, Kam Yim Sze, and Ka-Wai Kwok are with the Department of Mechanical Engineering, The University of Hong Kong, Hong Kong; (Tel: +852-2219-4494; Fax: +852-2547-4699; e-mail: kwokkw@hku.hk)

${ }^{2} \mathrm{~W}$. Luk are with the Department of Computing, Imperial College London, SW7 2AZ, U.K.

${ }^{3}$ C.K. Yeung is with Bio-Medical Engineering (HK) Limited, and Department of Surgery, Li Ka Shing Faculty of Medicine, The University of Hong Kong, Hong Kong. analytical model for such robot designs. Moreover, the model may be too specific to the robot design, not capable to be applied for modular structure, even though each robot module may be made of the same materials. Much effort is paid for determining the parameters/coefficients involved in the model, as to which could be a heuristic search accomplished by trial-and-error. Impractical assumptions is usually taken account to reduce the complexity of this search, for example, the constant curvature is assumed in the work of segmented continuum robot [6,7]; however, this usually violates in situations when the robot encounters the unevenly force distribution, substantially degrading the model reliability in real applications.

Online estimation of robot motion mapping using machine intelligence methods solely, in absence of either kinematic, physical or analytical model, is also the state-ofthe-art for resolving the challenges in soft-robotic control. Such model-free approaches can be optimized and updated [11], even using reinforcement learning [12] or neural network [13]. Those continuum structures susceptible to external disturbance, but find their ways impossible to exploit any physical/analytical model, e.g. robotic catheters, will make use of these model-free approaches, however, which are prone to keeping inputs with sufficient number of training samples.

Finite element modelling (FEM) is a powerful numerical approach to perform piecewise approximation continuously with the prior knowledge of material properties given. It acts as an ultimate solution to describing any mechanical structure. Recent work demonstrated that FEM is capable of performing kinematic analysis on soft robot constituted with inhomogeneous composite materials, although the deformation involved is yet sufficiently large to provide a full range of robot motion [14-17].

In this paper, we propose to develop a generic control framework for soft robotic manipulation, of which the forward kinematics can be characterized by hyper-elastic FEM $[18,19]$. Detailed volumetric discretization is applied to model our fabricated soft tentacle driven by three fluidic chambers under accurate pressure-feedback control. The resultant model's reliability and accuracy enable the teleoperated control of our fabricated robot to perform largedeformation manipulation for intracavitary navigation. The major contributions of this work can be listed as follows:

- Our tele-operated control framework provides a proofof-concept of incorporating the FEM into a feedforward control of a soft continuum robot; 
- Motion parameterization is applied to describe the motion transition pre-computed by the FEM throughout the soft robotic workspace;

- Experimental validation is conducted, allowing for accurate and consistent path-following manipulation with the fully used soft robot workspace.

\section{FINITE ELEMENT ANALYSIS MODEL DESIGN}

The finite element model of a flexible compliant manipulator composed of soft and incompressible elastomer is presented as shown in Fig. 1. This manipulator consist of three inflatable chambers. Each chamber is constrained by strain-wrapping in transversal plane in order to attain anisotropic expansion along axial direction upon inflation of pressurized fluid. Incorporating such elongation by carefully regulating the inflation pressure of each chamber will achieve a full paranoiac motion. In this paper, we employ FEA as the major cue to characterize such kinematics mapping from pressure actuation to manipulator motion.

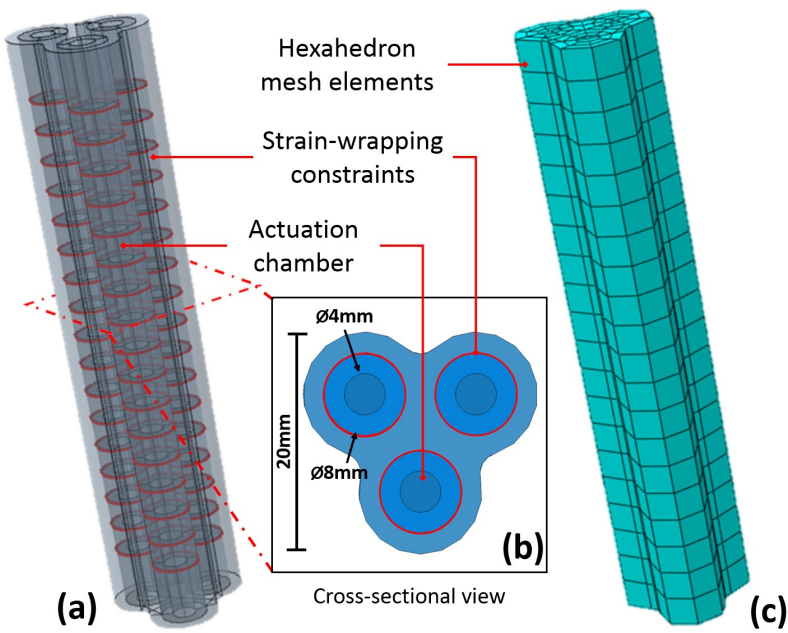

Fig. 1. $C A D / C A M$ model of the soft manipulator showing (a) strainwrapping constrains around individual actuation channel to achieve anisotropic expansion; (b) cross-sectional view of the manipulator; (c) finite element mesh tessellated with 1178 linear hexahedron elements. A set of 408 truss elements are binded to the nodes directly to emulate the effect of strain-wrapping constraint.

The hyper-elastic incompressible property of silicone rubber is modeled by Neo Hookean model [20] in this analysis. The strain energy equation is stated as follow:

$$
\Psi=c_{1}\left(\bar{I}_{1}-3\right)
$$

where shear modulus $\mu=2 c_{1}, \bar{I}_{1}$ is the first deviatoric strain invariant.

The manipulator model is meshed with 1178 hybrid linear hexahedral elements and 408 linear truss elements. Truss elements are chosen over beam elements to model the strain-wrapping constraint because it does not hinder bending. Hexahedral element is more preferable than tetrahedral element due to the fact that hexahedral element can better resemble the hyper-elastic stress-strain relationship of realistic material under large deformation [20]. Hexahedral element is, in general, more difficult to tessellate complicated volumetric geometry, such as circles and sharp edges, compared to tetrahedral element.
Despite of its complexity in meshing, it can fill up the same volume with smaller number of elements, resulting in reduced computational requirement.

After proper meshing, different external loads including gravity and inflation are then applied to the finite element model for further evaluation to estimate the kinematics mapping as described in Section III. Quasistatic motion is taken as an assumption in which the manipulator is expected to move at low velocities, and negligible hysteresis loss in operation.

\section{METHODOLOGY}

In this paper, we aim to demonstrate our proposed control framework by targeting the tele-operated soft manipulator to follow a pre-defined trajectory on the intracavity surface under human supervision. Such task is admissible to various robotic applications. For examples, surgical procedures that require precise targeting of highpower laser to perform tissue ablation or cutting; Full inspection of an intracavity space using endoscopic camera view through a desired pathway.

\section{A. Characterization of robot kinematics in intracavity space}

Finite element analysis is employed to characterize the robot configuration when chambers are actuated by pressurized fluid. A set of inflation pressure combination $U=\left\{u_{1}, u_{2}, \ldots, u_{M}\right\}, u_{i} \in \mathbb{R}^{N}$ is constructed by sampling from the feasible actuation space, where $N$ is the number of inflatable chambers and $M$ is the total number of simulations performed. This sampling interval could be a flexible function depending on the kinematic complexity and significance of the neighbor region. These sets of pressure input are then applied as distributed load on the robot FEA model to simulate the corresponding deformation. For our soft robotic manipulator (Fig. 1), a total number of 961 simulations $(M=961)$ are performed to find out the deformation characteristics as shown in Fig. 6. We define the discrete mapping between actuation input and resultant end-effector position as a set of discrete mapping pairs:

$$
F=\left\{\left({ }^{w} x_{1}, u_{1}\right),\left({ }^{w} x_{2}, u_{2}\right), \ldots,\left({ }^{w} x_{M}, u_{M}\right)\right\}
$$

where ${ }^{w} x_{i}=\left[p_{i}, n_{i}\right]^{T} \in \mathbb{R}^{6}$ is the $6 \mathrm{D}$ position and orientation of manipulator end-effector after inflation and the super-script refers to world coordinate.

To navigate the targeting point ${ }^{w} s_{i} \in \mathbb{R}^{3}$ of end-effector smoothly on the intracavity task space $S$, all of the deformation mapping are transformed into the task space $S$ (Fig. 2). This is done by finding the intersection between the principle axis of manipulator end-effector and the intracavity surface $S$.

$$
{ }^{w} s_{i}=\operatorname{Intersection}\left(S,{ }^{w} x_{i}\right) \text {, for } i=i \ldots M
$$




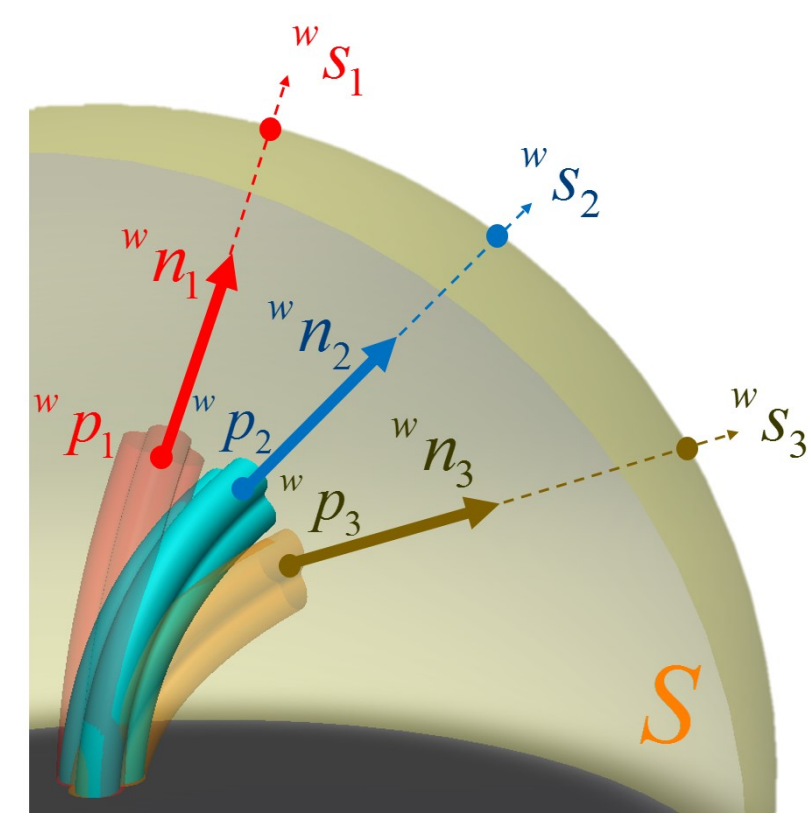

Fig. 2. The soft robot configurations under various inflation pressure is simulated by FEA. The corresponding targeting point ${ }^{w} S_{i}$ on intracavity surface $S$ (yellow) is the extension of the manipulator end-effector at ${ }^{w} p_{i}$ along the normal ${ }^{w} n_{i}$.

\section{B. Local linearization of robot kinematics model}

To provide intuitive human-robot interaction during navigation, we have established a reliable visual guidance that accommodate visual-motor re-alignment. It is achieved by attaching a "virtual camera coordinate" $L$ at the endeffector coordinate with its principle axis aligned with its Z-axis (Fig. 3). A virtual camera view is then rendered to visualize the pre-defined trajectory and intracavity surface $S$ in the image coordinate. This visual guidance realign the visual-motor mapping of Jacobian matrix and hence enhance the hand-eye coordinate of operators. As they are allowed to issue desired incremental end-effector motion in the planar image coordinate, which simplifies the control complexity by reducing the required control input into a two dimensional vector $\Delta y \in \mathbb{R}^{2}$ in image plane.

The kinematics model of the soft manipulator with respect to the camera coordinate is identified by best fitting a linear function to the neighbour discrete kinematics mapping pairs $F$ pre-computed by FEM simulation. Following the concept of virtual camera coordinate, a mapping pair $F_{i}$ is considered as viable neighbourhood and selected for linear fitting only if it lies within a kinematics field of view (FOV) of the camera coordinate $L$ (Fig. 3). This FOV is not to be confused with the visualization rendering FOV of the virtual camera visual guidance, where the kinematics FOV is chosen to accommodate a locally viable kinematics model.

As illustrated in Fig. 3, assuming that a mapping sample point $F_{j}\left({ }^{w} x_{j}, u_{j}\right) \in F$ lay within the kinematics FOV of the current camera coordinate $L,{ }^{w} x_{j}$ is projected on the image plane of $L$ as ${ }^{c} y_{j}=\left[d_{x}, d_{y}\right]^{T} \in \mathbb{R}^{2}$. A set of local kinematics mapping $H=\left\{\left({ }^{c} y_{j}, \Delta u_{j}\right)\right\}, j=1, \ldots, h$, $h>n$ can hence be obtained by projecting all neighbour ${ }^{w} s_{j}$ on the image plane and differencing the control pressure ${ }^{c} u_{j}$ with current pressure, where $\Delta u_{j}=u_{j}-u_{\text {current }}$. One can imagine this kinematics mapping as the end-effector displacement ${ }^{c} \Delta y_{j}$ in image coordinate $L$ corresponding to the change of actuation pressure $\Delta u_{j}$. The linear kinematics model can be formulated as a Jacobian matrix that linearly map the actuation input to targeting point displacement ${ }^{c} \Delta y \quad$ w.r.t. the origin of image plane:

$$
{ }^{c} \Delta y=J \Delta u
$$

Each row of the Jacobian matrix is found by least square fitting method as formulated below at every control iteration.

$$
\min _{J_{1}} \frac{1}{2}\left\|\left[\begin{array}{c}
u_{0}^{T} \\
u_{1}^{T} \\
\vdots \\
u_{h}^{T}
\end{array}\right] J_{1}^{T}-\left[\begin{array}{c}
d_{x 1} \\
d_{x 2} \\
\vdots \\
d_{x h}
\end{array}\right]\right\|_{2}^{2}, \min _{J_{2}} \frac{1}{2}\left\|\left[\begin{array}{c}
u_{0}^{T} \\
u_{1}^{T} \\
\vdots \\
u_{h}^{T}
\end{array}\right] J_{2}^{T}-\left[\begin{array}{c}
d_{y 1} \\
d_{y 2} \\
\vdots \\
d_{y h}
\end{array}\right]\right\|_{2}^{2}
$$

where $J=\left[\begin{array}{l}J_{1} \\ J_{2}\end{array}\right], J_{i} \in \mathbb{R}^{1 \times n}$ and $\|\cdot\|_{2}$ is the L-2 norm operator for vector.
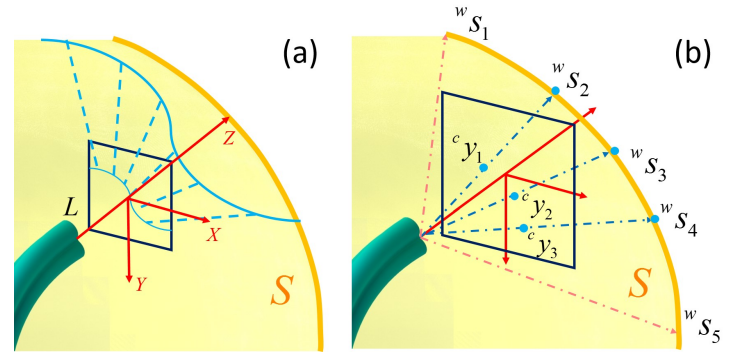

Fig. 3 (a) Illustration of the "virtual camera view" provided to the operators during tele-operative navigation. The Jacobian matrix of motion mapping is estimated w.r.t. this image coordinate, hence aligned with the visual guidance. (b) The neighbor targeting points $\left({ }^{w} S_{2},{ }^{w} S_{3},{ }^{w} S_{4}\right)$ on intracavity surface $S$ (yellow) are projected on the camera coordinate $L$ (red) as ${ }^{w} y_{i}$ which are used for local kinematics linearization. Others targeting points $\left({ }^{w} S_{1},{ }^{w} S_{5}\right)$ are neglect as they are out of the kinematics FOV.

\section{Optimization-based Inverse kinematics solver}

With the estimated Jacobian matrix, it is possible to resolve the inverse kinematics for the required actuation $\Delta u$ to attain the desired image plane displacement ${ }^{c} \Delta y$ as specified by the operator. However, such system of linear equations might not have a close form solution due to the fact that Jacobian matrix does not have full rank in general. For the case of our soft manipulator, three chambers can be actuated to achieve two dimensional desired motion in image coordinate, which give infinitely many solutions. This under-defined equation system also yield additional flexibility to select preferable robot behavior, such as minimizing the actuation effort or total inflation pressure to enhance robot stability.

This inverse kinematics problem is formulated as a constrained optimization problem to search for the most appropriate actuation. The cost function is defined as the sum of actuation pressure change in order to minimize the resultant pressure of each chamber to avoid fatigue of elastomer due to high inflation pressure and expansion. 


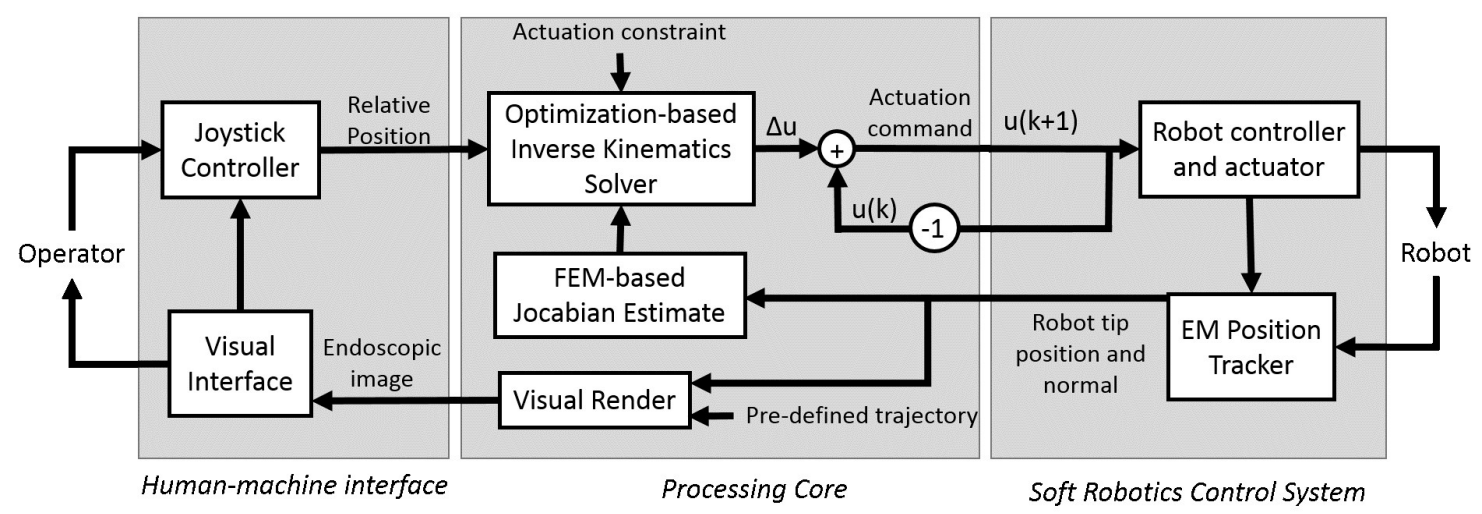

Fig. 4. System architecture of the experimental setup illustrating the interconnection of the primary components: the human-machine interface, the process core that implement our proposed soft robot control framework and the robot control system.

subject to:

$$
\min _{u} \sum_{m} \Delta u_{i}
$$

$$
\begin{array}{ccc}
J \Delta u & = & { }^{c} \Delta y_{\text {desired }} \\
u_{\text {current }}+\Delta u & \geq 0 \\
u_{\text {current }}+\Delta u & \leq & u_{\max }
\end{array}
$$

The linear kinematics model using Jacobian matrix is defined as a part of the constraint in this optimization problem. Other constraints include limitations on the inflation pressure above zero, because negative pressure is not allowed in our volumetric control. The pressure change is also constrained to prevent individual chamber from over inflation. Similar constraints may also apply to other actuation mechanism, such as tendon-driven actuators. This constrained convex optimization is resolved efficiently using interior point method in each control iteration. The optimal solution will be used to update the actuation input of soft robot.

$$
u[k+1]=u[k]+\Delta u
$$

\section{EXPERIMENTAL SETUP}

A robot identical to the design shown in Fig. 1 is fabricated using Ecoflex 0050 (Smooth-on inc.) to verify the control algorithm. Three individual actuation chambers, with $4 \mathrm{~mm}$ internal diameter and $8 \mathrm{~mm}$ outer diameter, are molded separately. Kevlar strings are wrapped in single helical structure and used as strain-wrapping constraints. Three wrapped chambers are glued into a single soft manipulator by a secondary molding layer of Ecoflex 0050 . The soft manipulator was actuated via pneumatic volumetric control system.

Block diagram of the overall control system, including human-machine interface, processing core and soft robotic manipulation system, is illustrated in Fig. 4.

Soft manipulation tentacle is actuated by three separable power-screw fluidic cylinders with volumetric control. A volumetric control system is implemented for the setup. During the experiment, the particular combination of pressures of three separate chambers are actuated and the manipulator is driven to the designated position.

The soft robot manipulator is controlled via a LWPRbased system. Before operation, data are collected from real experiment to train the system. The data contain the mapping between the chambers' pressure and the position and orientation of the end-effector, which is recorded by an electro-magnetic (EM) tracking coil mounted on the top of soft robot manipulator. After the system training, the current position of the soft manipulator end-effector is continuously recorded and used for the Jacobian Estimation and to provide a visual feedback to the operator. By inputting a target position, difference between it and current position can be calculated and then the change in pressure for the actuation chambers can be estimated by an optimization-based inverse kinematics solver to actuate the end-effector.

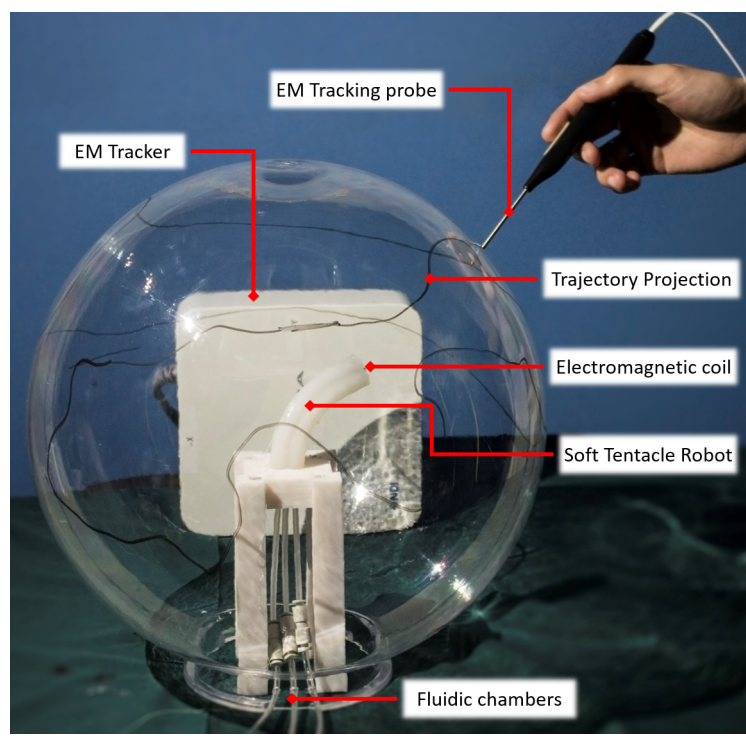

Fig. 5. The desired trajectory is pre-registered on the intracavity surface (transparent sphere shell) by using EM position tracking system. The soft manipulator is controlled by operator's joystick input to trace the trajectory in virtual camera perspective. The position of the end-effector is also monitored by the same EM system throughout the experiment to validate the trajectory tracking accuracy of the proposed control framework.

The soft robotic manipulator is placed inside a transparent spherical shell with $30 \mathrm{~cm}$ diameter so as to stimulate a $3 \mathrm{D}$ workspace. A pre-defined trajectory is registered using with an EM tracking probe, and stored as a tracking target for the manipulator as shown in Fig. 5. 
Throughout the experiment, the trajectory of figureeight shape is separated into a series of points, continuously updated as target positions of the manipulator. The endeffector is then led by the trajectory for a certain amount of loops. By comparing the target trajectory with the end effector position and orientation measured by EM Tracking system, the accuracy and stability of the control scheme can be evaluated.

\section{RESULTS AND DISCUSSION}

Results of FEA simulation showing the bending angles and steering angles upon inflation is presented in Fig. 6 below. The comparison of simulation results and the real model is also presented in Fig. 7. As mentioned in the section II, actuation effect of each individual chamber is complementary to the combined effect of other two. As the model is rotationally symmetric, the sample points for the determination of the kinematics in the whole paranoiac workspace can be collected by FE simulation of inflating two actuation chambers only.

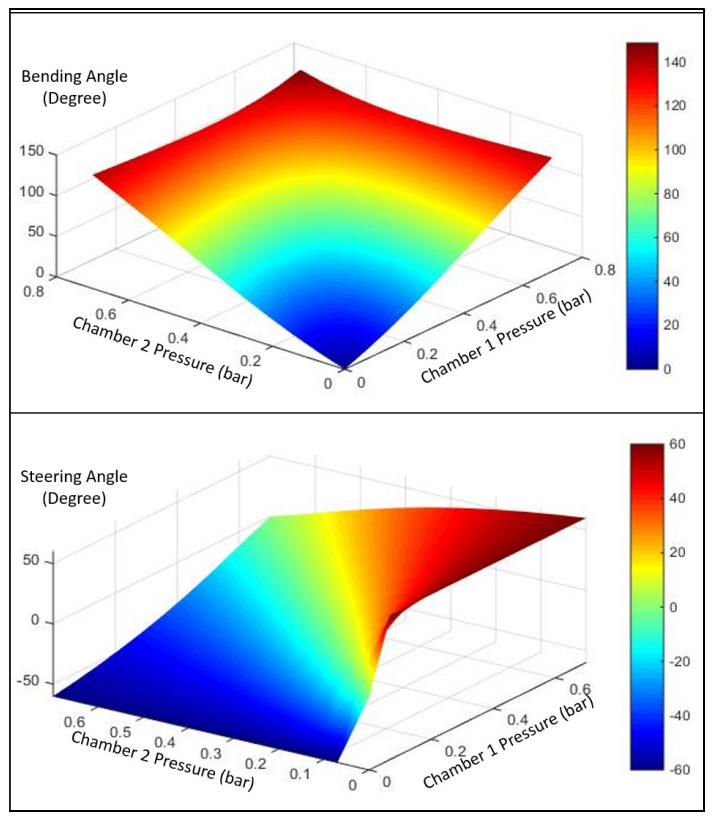

Fig. 6. Illustration of the bending angle (upper) and the steering angle (lower) of the soft robot under different pressure applied on the actuation chambers determined by finite element analysis.

The pre-defined helical target trajectory and the trajectory of the intersection point of principle axis of the virtual camera and the spherical intracavity workspace is presented in Fig. 8. The error is defined as the distance between the pointing position and the designated position on the target trajectory. The error of the control is also presented in Fig. 9.

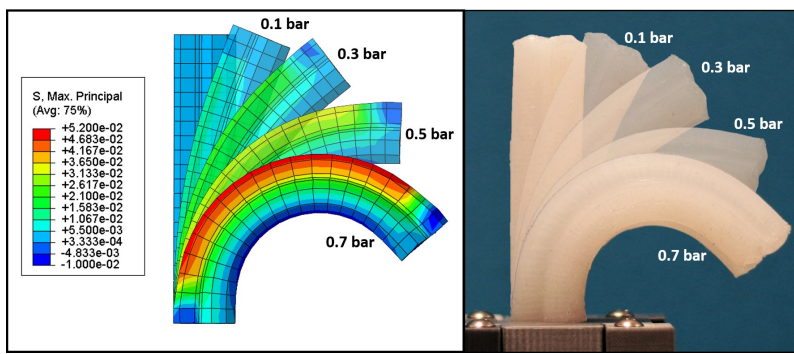

Fig. 7. Fine-tuned FEM model (right) demonstrate similar deformation characteristics to real soft manipluator (left) under various inflation pressure.

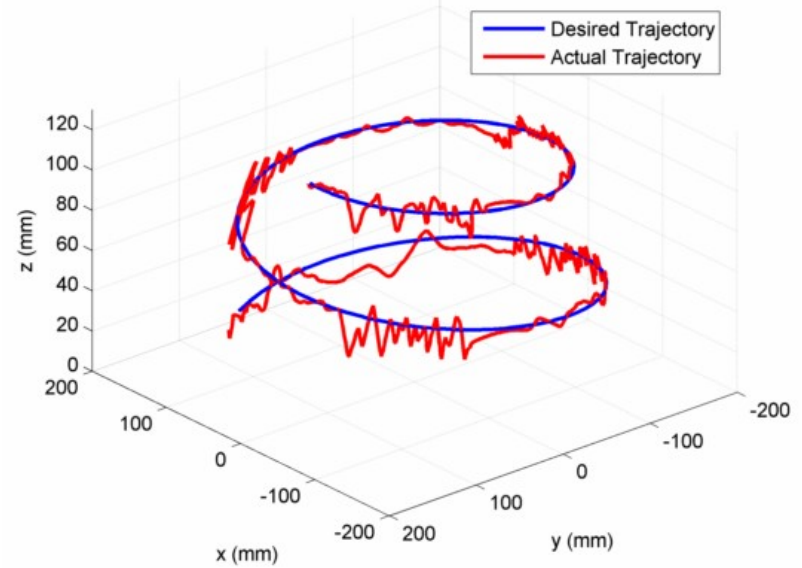

Fig. 8. Results from the trajectory tracking experiment. Blue line represents the pre-definied taregt trajectory. Red line represents the resultanlt trajectory of the targeting point of manipulator end-effector projected on the spherical intercavity surface.

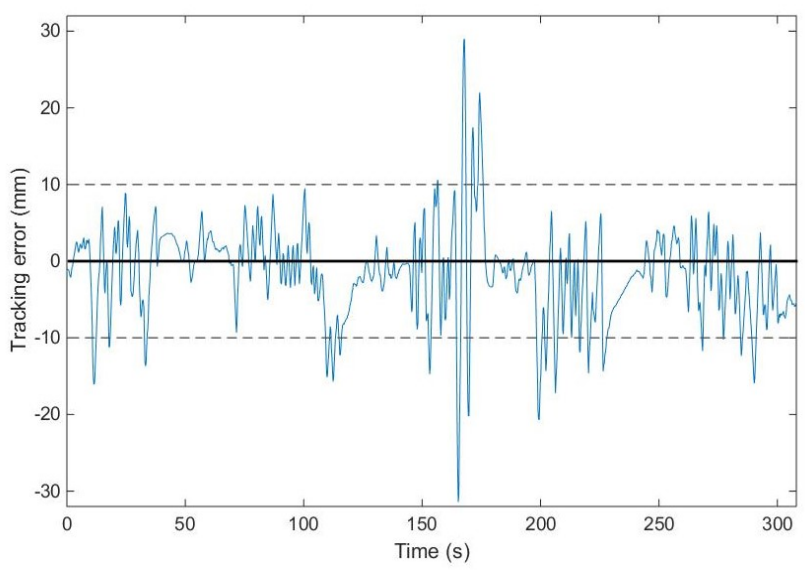

Fig. 9. Absolute error of the tracking result in millimeters.

It is worth to mention that most of the error of the end effect is within the range of $\pm 10 \mathrm{~mm}$, and the standard deviation of the error is below $6 \mathrm{~mm}$. The small error indicates that the FEM based control framework can be used to reliably estimate the Jacobian. The optimizationbased inverse kinematics estimation can also provide reliable actuation for the soft robot.

In this work, a virtual camera is adopted for the ease of user's control. By adopting the virtual camera and use the virtual image coordinate as an input, the system can help the user to perceive the $3 \mathrm{D}$ geometry more conveniently. Instead of requiring the user to map the input and the movement, the virtual camera control can help the user to give control commands based on the $2 \mathrm{D}$ image plane for the user's intuitive control.

The concept of reduced dimensionality using the image coordinate can also be applied on soft robotics with more complex structures and/or with multiple segments. Theoretically, the FEM-based control can be used to control all kinds of soft robotics when sufficient data points is collected.

The current FEM-based control framework is largely limited by its computational requirement. Several tricks were done in an attempt to cut down the computation. The current control framework attempted to make use of precomputed FEA results to obtain the kinematics of the robot. 
Quasi-static simulations and reduced integration is adopted to further cut down the computational requirement. However, with the current control framework, additional terms have to be included into the optimization model in order to deal with the robot's dynamics. For example, external contact and disturbance. The current control model have also neglected the effect of hysteresis of the softbodied manipulator. Explicit FEA may be a solution for the dynamics problem as it can effectively simulate dynamics problem, but performing explicit FEA for the control framework will require additional computation requirement.

\section{CONCLUSION AND FUTURE WORK}

This work has opened a new perspective to make use of FEM for controlling a soft robot with hyper-elastic properties. The time-to-time estimation of robot Jacobian could be altered by the pre-computed results of FEM. Experimental validation has been realized with our fabricated soft continuum robot, showing the promising path following via the proposed human-control interface. Future work will be first focused on resolving the computational bottleneck of hyper-elastic FEM through the implementation of high-performance computing (HPC) architecture. This will incorporate not only parallel, but also reconfigurable computing features (found in FieldProgrammable Gate Arrays), in order to pinpoint the complexity with regard to large memory access inevitably induced by numerous boundary problems.

Such computation could be incorporated into a separated feedforward control loop running at relatively low frequency. This work has been a good proof-ofconcept to demonstrate the advantage of decoupling the real-time Jacobian estimation from the FEM if that can be even updated online. It is anticipated that this prospective online FEM could give strong cues in regards of any interaction with the external disturbance.

\section{ACKNOWLEDGMENT}

This work is supported in parts by the Croucher Foundation, the Research Grants Council (RGC) of Hong Kong (Ref. No. 27209151 and No. 17227616), the Innovation and Technology Fund (ITF) of Hong Kong (ITS/361/15FX), NISI (HK) Limited, the European Horizon 2020 Research and Innovation Programme under grant agreement number 671653, and the UK EPSRC (EP/L00058X/1， EP/L016796/1， EP/N031768/1 and EP/P010040/1), Intel, Maxeler, and Xilinx.

\section{REFERENCES}

[1] D. Trivedi, C. D. Rahn, W. M. Kier, and I. D. Walker, "Sof robotics: Biological inspiration, state of the art, and future research," Applied Bionics and Biomechanics, vol. 5, pp. 99-117, 2008.

[2] S. Mao, E. Dong, H. Jin, M. Xu, S. Zhang, J. Yang, et al., "Gait study and pattern generation of a starfish-like soft robot with flexible rays actuated by SMAs," Journal of Bionic Engineering, vol. 11, pp. 400-411, 2014.

[3] S. Sareh, A. Jiang, A. Faragasso, Y. Noh, T. Nanayakkara, P. Dasgupta, et al., "Bio-inspired tactile sensor sleeve for surgical soft manipulators," in IEEE International Conference on Robotics and Automation (ICRA), 2014, pp. 1454-1459.

[4] F. Maghooa, A. Stilli, Y. Noh, K. Althoefer, and H. A Wurdemann, "Tendon and pressure actuation for a bio-inspired manipulator based on an antagonistic principle," in IEEE
International Conference on Robotics and Automation (ICRA), 2015, pp. 2556-2561.

[5] M. Cianchetti, T. Ranzani, G. Gerboni, T. Nanayakkara, K Althoefer, P. Dasgupta, et al., "Soft robotics technologies to address shortcomings in today's minimally invasive surgery: the STIFFFLOP approach," Soft Robotics, vol. 1, pp. 122-131, 2014.

[6] D. B. Camarillo, C. F. Milne, C. R. Carlson, M. R. Zinn, and J. K. Salisbury, "Mechanics modeling of tendon-driven continuum manipulators," IEEE Transactions on Robotics, vol. 24, pp. 1262 $1273,2008$.

[7] B. Jones and I. D. Walker, "Kinematics for multisection continuum robots," IEEE Transactions on Robotics, vol. 22, pp. 43-55, 2006.

[8] M. Mahvash and P. E. Dupont, "Stiffness control of surgical continuum manipulators," IEEE Transactions on Robotics, vol. 27, pp. 334-345, 2011

[9] R. J. Webster and B. A. Jones, "Design and kinematic modeling of constant curvature continuum robots: A review," The International Journal of Robotics Research, 2010.

[10] Y. Ganji and F. Janabi-Sharifi, "Catheter kinematics for intracardiac navigation," IEEE Transactions on Biomedical Engineering, vol. 56, pp. 621-632, 2009.

[11] M. C. Yip and D. B. Camarillo, "Model-less feedback control of continuum manipulators in constrained environments," IEEE Transactions on Robotics, vol. 30, pp. 880-889, 2014.

[12] A. Rost and S. Schadle, "The SLS-Generated Soft Robotic HandAn Integrated Approach Using Additive Manufacturing and Reinforcement Learning," in 12th International Conference on Machine Learning and Applications (ICMLA), 2013, pp. 215-220.

[13] M. Giorelli, F. Renda, M. Calisti, A. Arienti, G. Ferri, and C. Laschi, "Neural Network and Jacobian Method for Solving the Inverse Statics of a Cable-Driven Soft Arm With Nonconstant Curvature," IEEE Transactions on Robotics, vol. 31, pp. 823-834, 2015.

[14] I. D. Walker, "Continuous backbone "continuum" robot manipulators," ISRN Robotics, 2013.

[15] C. Duriez, "Control of elastic soft robots based on real-time finite element method," in IEEE International Conference on Robotics and Automation (ICRA), 2013, pp. 3982-3987.

[16] F. Largilliere, V. Verona, E. Coevoet, M. Sanz-Lopez, J. Dequidt, and C. Duriez, "Real-time control of soft-robots using asynchronous finite element modeling," in IEEE International Conference on Robotics and Automation (ICRA), 2015, pp. 2550 2555.

[17] J. Bosman, T. M. Bieze, O. Lakhal, M. Sanz, R. Merzouki, and C. Duriez, "Domain decomposition approach for FEM quasistatic modeling and control of Continuum Robots with rigid vertebras," in IEEE International Conference on Robotics and Automation (ICRA), 2015, pp. 4373-4378.

[18] R. Ogden, G. Saccomandi, and I. Sgura, "Fitting hyperelastic models to experimental data," Computational Mechanics, vol. 34, pp. 484-502, 2004

[19] P. Martins, R. Natal Jorge, and A. Ferreira, "A Comparative Study of Several Material Models for Prediction of Hyperelastic Properties: Application to Silicone-Rubber and Soft Tissues," Strain, vol. 42, pp. 135-147, 2006.

[20] S. E. Benzley, E. Perry, K. Merkley, B. Clark, and G. Sjaardama, "A comparison of all hexagonal and all tetrahedral finite element meshes for elastic and elasto-plastic analysis," in Proceedings, 4th International Meshing Roundtable, 1995, pp. 179-191. 